\section{Insegurança Alimentar e estado nutricional de crianças de São João do Tigre, no semi-árido do Nordeste}

\section{Food insecurity and nutritional status of children of São João do Tigre, semi-arid area of Northeast Brazil}

Juliana Souza Oliveira'

Pedro Israel Cabral de Lira"

Sonia Lucia Lucena Sousa de Andrade"

Aline Cabral Sales"

Sandra Regina Maia"

Malaquias Batista Filho'"'

'Departamento de Nutrição da Universidade Federal de Pernambuco e da Universidade Federal de Alagoas.

" Departamento de Nutrição da Universidade Federal de Pernambuco.

III Instituto Materno Infantil Profo Fernando Figueira (IMIP) e Departamento de Nutrição da Universidade Federal de Pernambuco.

Financiamento: Conselho Nacional de Desenvolvimento Científico e Tecnológico - CNPq (processos no 502955/2003-1 e 505808/2004-8 e bolsas de produtividade em pesquisa dos profs. Malaquias Batista Filho e Pedro Israel Cabral de Lira).

Agradecimentos: Ao CNPq pelo financiamento (Processos no. 502955/03-1 e 505808/04-8) e pela Bolsa de Produtividade em Pesquisa dos Profs. Malaquias Batista Filho e Pedro Lira, à nutricionista Ida Cristina (in memorian), aos Técnicos da Prefeitura de São João do Tigre, à equipe de campo e a todas as famílias pelo apoio e colaboração.

Correspondência: Juliana Souza Oliveira. Av. Boa Viagem, 6836, Bloco 18, Aptº 32, Boa Viagem Recife, PE - CEP 51130-000. E-mail: juliana_nutricao@yahoo.com.br

\section{Resumo}

Objetivo: Analisar o estado nutricional de menores de cinco anos e sua relação com a situação de (in)segurança alimentar. Métodos: Estudo transversal de 458 famílias, com 558 menores de 5 anos, realizado no município de São João do Tigre. A avaliação da (in)segurança alimentar foi realizada mediante aplicação da Escala Brasileira de Insegurança Alimentar (EBIA). A categorização do estado nutricional foi feita a partir dos indicadores estatura/idade, peso/idade e índice de massa corporal (IMC), utilizandose as curvas da Organização Mundial da Saúde. Também foram analisadas as associações com os indicadores socioeconômicos e biológicos da criança sobre o índice estatura/idade. Resultados: A segurança alimentar foi caracterizada em torno de $13 \%$ das famílias, prevalecendo nas demais a condição de insegurança alimentar, sendo a forma moderada a predominante $(40,2 \%)$. Encontrou-se uma frequência mais baixa do que o normalmente esperado de desnutrição pelo IMC $(1,2 \%$ e $1,3 \%$, respectivamente, para as áreas rural e urbana), com resultados bem mais elevados ao se considerar o índice estatura/idade (16,8\% e 12,9\%). A análise de regressão linear multivariada revelou que apenas duas variáveis (renda familiar per capita e escolaridade materna) influenciaram significativamente o índice estatura/idade. Conclusão: Em população de elevado grau de pobreza, baixo Índice de Desenvolvimento Humano e prevalência quase generalizada de insegurança alimentar, o uso exclusivo de indicadores antropométricos pode revelar uma situação aparentemente bem mais favorável. Portanto, os resultados não podem ser interpretados apenas no campo da validade interna, mas no interior de um processo de rápidas mudanças que caracterizam a transição nutricional em curso, atingindo inclusive as populações de condições de vida excepcionalmente desfavoráveis.

Palavras-chave: Insegurança alimentar. Pobreza. Estado nutricional. Crianças. 


\section{Abstract}

Objective: To analyze the nutritional status of children under five years of age and to verify its relation with food insecurity profile. Methods: This is a cross-sectional study of 458 families with 558 children in the city of São João do Tigre, semi-arid area of the state of Paraíba, Brazil. Food insecurity was determined based on the Brazilian Food Insecurity Scale. Nutritional status was assessed through height-forage, weight-for-age and Body Mass Index (BMI), using the World Health Organization curves as a reference. The associations of socioeconomic and biological variables with height-for-age were also examined. Results: Food security was found in only $13 \%$ of families, and a moderate insecurity condition was more prevalent $(40.2 \%)$. There was a lower frequency of malnutrition for BMI than normally expected $(1.2 \%$ and $1.3 \%$ respectively for the rural and urban areas) with a high prevalence when considering height-for-age indices $(16.8 \%$ and $12.9 \%$, respectively). The linear regression analysis showed that only two variables (per capita family income and maternal years of schooling) had a significant influence on child height-for-age. Conclusion: In a population with a high poverty degree, low Human Development Index and high prevalence of food insecurity, the exclusive use of anthropometric indicators may show a more favorable nutritional status. Therefore, the results cannot be interpreted only in the field of internal validity, but as part of a process of fast changes that characterize the nutritional transition in course, also reaching populations in unfavorable life conditions.

Keywords: Food insecurity. Poverty. Nutritional status. Children.

\section{Introdução}

A alimentação constitui um dos direitos humanos básicos, representando um corequisito para a legitimação de todos os outros direitos inerentes ao próprio conceito e exercício de cidadania. Abrange aspectos que não se limitam apenas ao acesso aos alimentos em si, estendendo-se ao próprio contexto em que pessoas e as comunidades estão inseridas, cabendo ao Estado a promoção e garantia de sua observação ${ }^{1-3}$, de modo a configurar uma prerrogativa essencial de todo cidadão de não sentir temor de viver sob a ameaça de fome $\mathrm{e}^{4}$.

Nesta perspectiva, o Brasil representa uma situação conflitiva, na medida em que, sendo um dos maiores produtores de alimentos do mundo, ainda convive com uma condição social em que milhões de pessoas se encontram à margem da cidadania, por não terem assegurado, entre outros, o direito básico à alimentação suficiente, completa, adequada e saudável ${ }^{4,5}$.

Resultando de fatores estruturais e conjunturais decorrentes da organização e funcionamento da sociedade, a insegurança de acesso a um elenco básico de alimentos de qualidade e em quantidades necessárias ou às condições para sua obtenção, constitui um desafio universal humano assumido pelas Nações Unidas há mais de três décadas ${ }^{6,7}$. As situações de insegurança alimentar podem ser identificadas por várias manifestações, como a fome, a desnutrição, as carências específicas, o excesso de peso, doenças geradas pela alimentação inadequada e consumo de produtos prejudiciais à saúde, como os contaminantes por agrotóxicos, metais pesados, fungos, bactérias e outros ${ }^{8,9}$.

Torna-se assim, oportuno e relevante identificar indicadores que possam verificar desde o direito de acesso aos alimentos até as condições concretas desse acesso e suas consequências finais no estado de saúde e nutrição dos indivíduos e coletividades. Segundo Segall-Côrrea ${ }^{8}$, (2007) nenhum indicador, isoladamente, consegue dar conta das múltiplas dimensões da 
segurança alimentar e nutricional (SAN), necessitando-se, portanto de instrumentos que possibilitem avaliar sua própria condição, em nível de populações, grupos sociais específicos, famílias e indivíduos, face à diversidade de exposições e de suas consequências ${ }^{10,11}$.

$\mathrm{Na}$ insegurança alimentar existem também componentes psicológicos importantes, como a preocupação, a incerteza de ter ou não alimento no próximo mês ${ }^{12}$. Nessa perspectiva, a partir da experiência da Universidade Cornell ${ }^{13-15}$ foi desenvolvido e validado, recentemente, um modelo de questionário voltado para a realidade brasileira, a Escala Brasileira de Insegurança Brasileira (EBIA) ${ }^{16}$, onde a questão alimentar é percebida em seus vários níveis: a preocupação de faltar alimento, o comprometimento da qualidade da alimentação da família e, como aspecto mais grave, a restrição quantitativa na disponibilidade de alimentos ${ }^{8,17}$.

A fome crônica, silenciosa, talvez seja a forma de carência mais difícil de ser identificada, mas nem por isso torna-se menos relevante. É um fenômeno que possui elementos socioeconômicos e culturais: insatisfeita, prolongada ou apenas parcialmente saciada, cria vulnerabilidades e muitas vezes se traduz em importantes patologias ${ }^{4,5,18}$.

O grupo populacional mais vulnerável à insegurança alimentar são as crianças, visto que as consequências nutricionais são mais imediatas e graves, e também porque, quando uma criança apresenta problemas no estado nutricional por insuficiência alimentar, pode-se conjecturar que no domicílio os adultos estejam se alimentando de maneira insuficiente há algum tempo ${ }^{19,20}$.

Com esta justificativa, o presente estudo tem como objetivo analisar, numa localidade intencionalmente estudada por seu acentuado grau de pobreza, com baixo índice de desenvolvimento humano (IDH), o estado nutricional de menores de 5 anos e sua relação com a situação de (in)segurança alimentar das famílias.

\section{Metodologia}

O estudo, do tipo transversal, foi realizado no período de maio a junho de 2005 . A população estudada consistiu numa amostra de 458 famílias com 558 menores de 5 anos (302 e 256 da zona urbana e rural, respectivamente) no município de São João do Tigre, micro-região dos Cariris Velhos, no Estado da Paraíba. Esta localidade foi selecionada intencionalmente, devido ao seu baixo Índice de Desenvolvimento Humano (IDH abaixo do $5^{\circ} \mathrm{P}$ ), estreita vinculação econômica e social entre a vida no campo e na cidade e, em razão das precárias condições socioeconômicas, riscos potenciais para as crianças menores de 5 anos e suas famílias.

Para o cálculo da amostra levou-se em consideração a prevalência de $60 \%$ de insegurança alimentar para o Nordeste, com base nos resultados da Pesquisa Nacional por Amostra de Domicílios - 2004 $4^{17}$, considerando um erro de $\pm 5 \%$, para um nível de significância de 95\%, que, acrescido de $10 \%$ para possíveis perdas, resultou numa amostra mínima de 440 famílias. Para o cálculo utilizou-se o programa STATCALC do EPI-INFO, versão, 6.04.

Foi usado instrumento de coleta de dados que inclui questionários para avaliação das informações.

Fez-se um estudo piloto com 30 famílias, objetivando, além de testar o instrumento de coleta, experimentar e ajustar a logística do trabalho de campo. $\mathrm{O}$ acesso ao domicílio foi realizado com a concordância do chefe da família. Para a avaliação antropométrica foi observado o consentimento livre e esclarecido de cada um dos membros da família, incluindo o consentimento do pai ou responsável para os menores de idade.

A avaliação da (in)segurança alimentar foi efetuada mediante a aplicação da Escala Brasileira de Insegurança Alimentar - EBIA $^{16}$, composta de 15 perguntas fechadas, com respostas positivas e negativas, relativas à percepção dos entrevistados sobre a situação alimentar vivida nos últimos três meses anteriores à entrevista. As 
respostas foram codificadas, considerando o processo de insegurança alimentar crescente, passando pela preocupação com a falta de alimentos, pelo comprometimento da qualidade da alimentação e, por último, pela deficiência quantitativa da alimentação e até mesmo fome entre adultos e crianças da família. Para as respostas positivas, foi atribuído o valor 1 (um) e, para as negativas, o valor 0 (zero), resultando num escore com amplitude teórica de 0 a 15 pontos. A soma dos escores resultantes foi classificada em quatro níveis: 0 (zero), segurança alimentar; 1 a 5, insegurança alimentar leve; 6 a 10, insegurança alimentar moderada; e 11 a 15, insegurança alimentar grave. Cada pergunta central era complementada por uma pergunta sobre a frequência com que o evento afirmativo ocorre. No presente estudo essas informações complementares sobre frequências não foram utilizadas.

A avaliação antropométrica foi realizada após treinamento dos pesquisadores segundo procedimentos técnicos recomendados pela Organização Mundial de Saúde $^{21}$ (1995). O peso corporal foi obtido utilizando-se balança digital (Filizola E150/3P, São Paulo, Brasil) com capacidade para $150 \mathrm{~kg}$ e precisão de 100g. Crianças que ainda não andavam foram pesadas nos braços do acompanhante, cujo peso individual foi deduzido do peso total do par acompanhante e criança. Os menores de dois anos foram medidos em decúbito dorsal, com as pernas juntas e em extensão (tomada do comprimento), utilizando o infantômetro (Raven Equipment, fabricado na Inglaterra) com amplitude de 100 $\mathrm{cm}$ e subdivisões de $0,1 \mathrm{~cm}$. As crianças maiores de dois anos foram medidas em pé e descalças, utilizando-se estadiômetro (Cardiomed, Curitiba-PR) com amplitude de $200 \mathrm{~cm}$ e subdivisões de $0,1 \mathrm{~cm}$.

Para garantir a acurácia das mensurações, as medidas foram tomadas duas vezes para cada criança, com a condição de que a diferença entre as avaliações não excedesse $0,5 \mathrm{~cm}$. Se este limite fosse eventualmente ultrapassado, repetia-se a mensuração, anotando-se as duas medições com valores mais próximos, utilizando sua média para efeito de registro.

Os questionários foram compostos por perguntas pré-codificadas e checadas diariamente para a identificação de possíveis erros de preenchimento. Para a validação da digitação, foi realizada a dupla entrada de dados, através do programa Epi Info, versão 6,04 (CDC, Atlanta, USA). As análises estatísticas foram realizadas através do software Statistical Package for the Social Sciences, version 12.0.1 for Windows (SPSS Inc., Chicago, IL, USA). Para análise da consistência interna das variáveis, foi calculado o Alpha de Cronbach, aceitando-se como satisfatório um valor mínimo de $0,85^{16}$.

$\mathrm{Na}$ avaliação do estado nutricional foi utilizado o software Anthro - $2007^{22}$, sendo as crianças classificadas pelos índices estatura/idade (E/I), peso/idade (P/I) e índice de massa corporal (IMC), expressos em escore-Z. O padrão de referência para classificação das medidas de peso e estatura foi o recomendado pela Organização Mundial da Saúde $^{23}$ (2006) e pelo Ministério da Saúde ${ }^{24}$ (2008), adotando-se os seguintes pontos de corte para categorização dos resultados: Desnutrição: <-2 escores Z; Risco nutricional: -2 escores $\mathrm{Z}$ a $<-1$ escore $\mathrm{Z}$; Adequado: -1 escore Z $\mathrm{a}<2$ escores Z; Sobrepeso: 2 escores Z.

A variável dependente estatura/idade também foi analisada como contínua. As associações estatísticas foram estimadas com referência a este índice, por representar a prevalência maior de déficit nutricional e ser considerada, atualmente, como a expressão epidemiológica mais característica e representativa de desvio antropométrico na população infantil brasileira ${ }^{25}$. O teste t-Student e a análise de variância (ANOVA) foram utilizados para comparar as diferenças entre médias nas análises bivariadas, considerando-se como significância estatística valores $p \leq 0,05$.

A matriz de correlação não apresentou multicolinearidade entre as variáveis, visto que os coeficientes de correlação de Pearson foram inferiores a 0,15 . Todas as variáveis com valor de $p<0,20$ na análise bivariada fo- 
ram selecionadas para a análise de regressão. As variáveis explanatórias eram dicotomas, exceto a (in)segurança alimentar, que foi tratada como variável dummy. A análise de regressão linear múltipla foi realizada utilizando-se o modelo hierarquizado, com a finalidade de avaliar o impacto das variáveis explanatórias sobre o índice estatura/idade.

Optou-se por um processo de modelagem de blocos, utilizando-se o método de sequência "enter", onde, primeiramente, o estado nutricional dos menores de cinco anos foi ajustado pela variável renda familiar per capita. O segundo bloco foi composto pelas variáveis escolaridade materna e localização geográfica. No terceiro bloco foi inserida a variável relacionada à (in)segurança alimentar. Finalmente, no quarto bloco, introduziu-se a idade da criança.

O projeto de estudo foi aprovado pelo Comitê de Ética do Instituto Materno Infantil Prof. Fernando Figueira - IMIP, no 386 , atendendo às normas regulamentares de pesquisas envolvendo seres humanos - Resolução 196/96 do Conselho Nacional de Saúde. Um médico pediatra fez parte da equipe de campo, com a responsabilidade de atender ou encaminhar para outros profissionais e serviços de assistência à saúde os casos clínicos que necessitassem de melhor exploração diagnóstica ou cuidados de acompanhamento imediatos ou sequenciais.

\section{Resultados}

O instrumento para avaliar a (in)segurança alimentar (escala EBIA) revelou uma consistência interna muito satisfatória, visto que o Alpha de Cronbach resultou em 0,91 para um mínimo aceitável de 0,85.

Verifica-se (Figura 1), que aproximadamente $13 \%$ das famílias dos menores de 5 anos do município foram classificadas na categoria de segurança alimentar, prevalecendo, assim, a condição de insegurança alimentar, sendo a forma moderada a predominante $(40,2 \%)$. Esta distribuição também ocorreu em relação à localização geográfica, zona urbana e rural, onde a situação mais prevalente foi a de insegurança alimentar moderada, com frequência de $38,4 \%$ e $42,3 \%$, respectivamente.

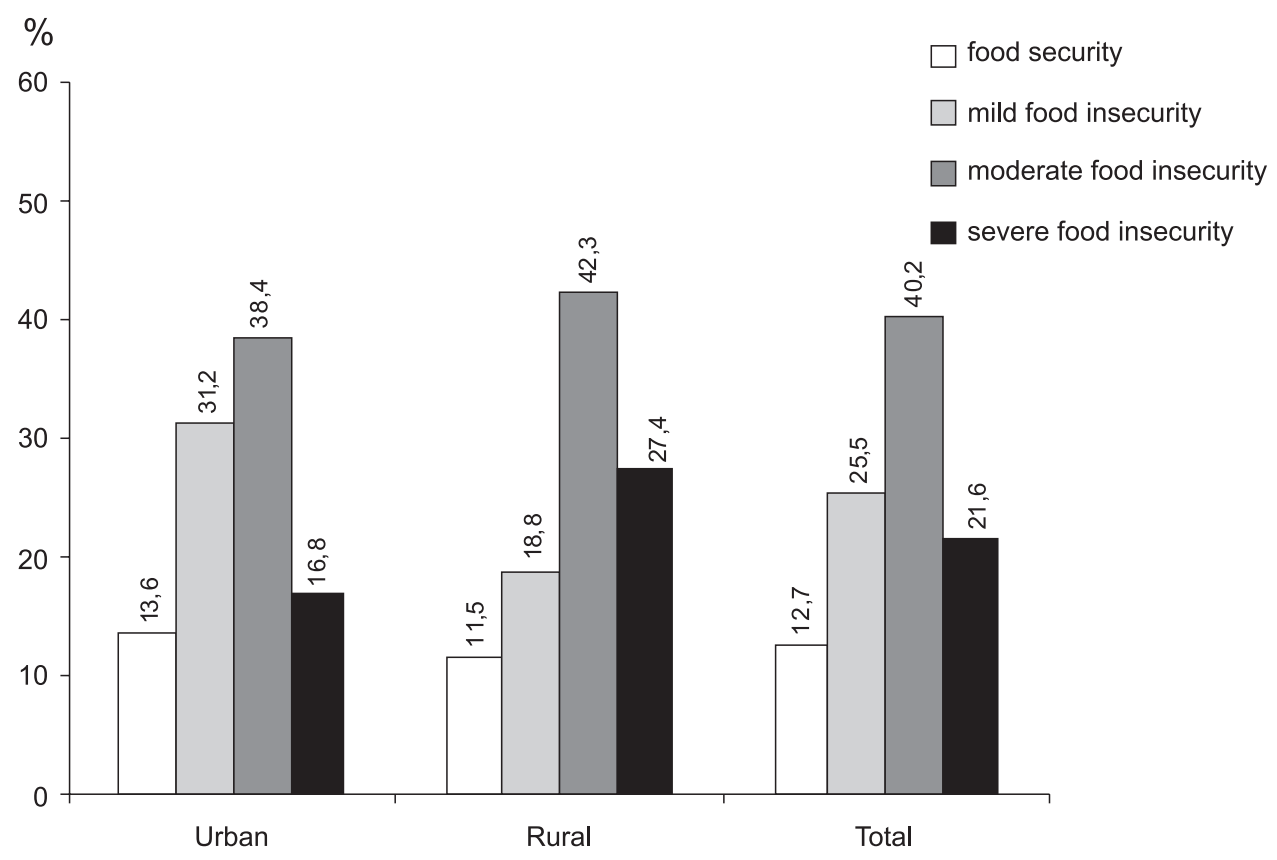

Figura 1 - Prevalência da (in)segurança alimentar das famílias de menores de 5 anos nas zonas urbana e rural do município de São João do Tigre - (PB), 2005.

Figure 1 - Prevalence of food (in)security in families with children under five years of age in urban and rural areas in the city of São João do Tigre - (PB), 2005. 
De acordo com a Figura 2, referente ao estado nutricional, verificou-se uma frequência muito baixa de desnutrição pelo índice de massa corporal (IMC), de $1,3 \%$ e $1,2 \%$, e de sobrepeso da ordem de $7,6 \%$ e $6,3 \%$ nas zonas urbana e rural, respectivamente, enquanto a prevalência de normalidade se situou em torno de $90 \%$. No que diz respeito ao índice peso/idade, obteve-se uma frequência de desnutrição de 4,0\% na área urbana e 6,6\% na rural. De acordo com o índice estatura/idade, foram verificadas elevadas prevalências de desnutrição nas duas áreas $12,9 \%$ na zona urbana e $16,8 \%$ na rural.

Como se demonstra na Tabela 1, 64,3\% dos menores de 5 anos se encontravam em famílias com condição de insegurança alimentar moderada ou grave. A (in) segurança alimentar, em seus diferentes graus de intensidade, quando relacionada ao índice estatura/idade, evidenciou uma associação estatisticamente significante, verificando-se que a média de escores $Z$ foi diminuindo à medida que a insegurança alimentar aumentava. No que diz respeito à localização geográfica, observou-se que a média de escores $\mathrm{Z}$ foi menor na zona rural, sendo a diferença estatisticamente significante. Nota-se que mais de $85 \%$ das famílias percebiam renda familiar per capita inferior a 0,5 salário mínimo, e que esta variável estava estatisticamente associada aos resultados do índice estatura/idade. A maior parte das mães $(57,3 \%)$ apresentava baixo nível de escolaridade, com predominância de casos com até 4 anos de estudo formal. Verificou-se que, à medida que aumentavam os anos de estudo, aumentavam as médias de escores Z. Quanto ao sexo e a idade das crianças, não houve diferença estatisticamente significante nas médias de escores $\mathrm{Z}$.

A análise dos efeitos ajustados das variáveis explicativas em relação ao índice estatura/idade encontra-se na Tabela 2. O primeiro modelo demonstra que a renda familiar per capita influenciou significativamente os resultados deste índice. O modelo 2 evidencia que a escolaridade

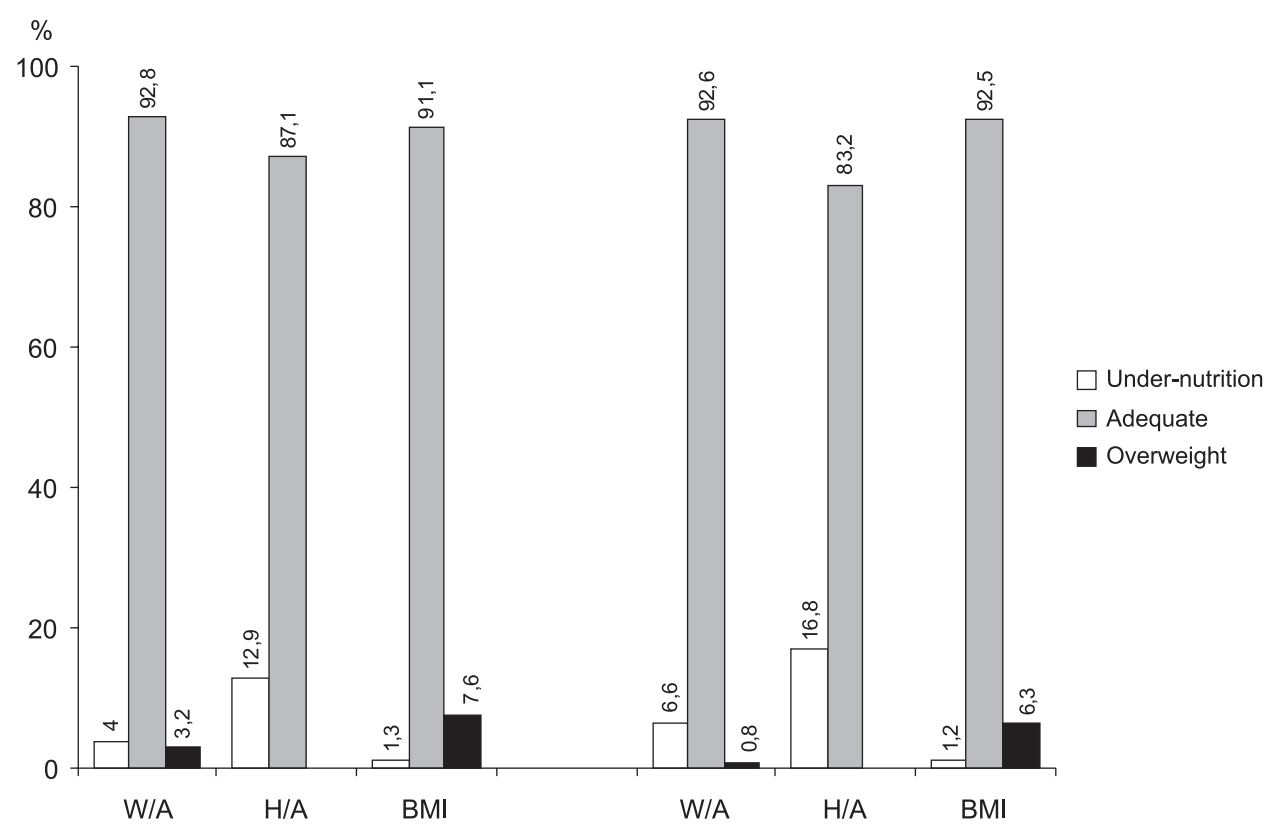

Figura 2 - Estado nutricional de menores de 5 anos das zonas urbano e rural de acordo com os indicadores peso/idade (P/I), estatura/idade (E/I) e índice de massa corporal (IMC). São João do Tigre - (PB), 2005.

Figure 2 - Nutritional status of children under five years of age in urban and rural areas according to weight-for-age (W/A), height-for-age (H/A) and Body Mass Index (BMI). São João do Tigre - (PB), 2005. 
Tabela 1 - Média do Índice estatura/idade (escore Z), de menores de 5 anos, segundo variáveis socioeconômicas e biológicas. São João do Tigre - (PB), 2005.

Table 1 - Mean of height-for-age index (Z-scores) in children under five years of age according to socioeconomic and biological variables. São João do Tigre - (PB), 2005.

\begin{tabular}{|c|c|c|c|c|c|}
\hline \multirow[t]{2}{*}{ VARIÁVEIS } & \multirow[b]{2}{*}{$\mathrm{N}$} & \multirow[b]{2}{*}{$\%$} & \multicolumn{3}{|c|}{ ESTATURA/IDADE } \\
\hline & & & Média & DP & $\mathrm{p}$ \\
\hline \multicolumn{6}{|l|}{ (In)segurança Alimentar } \\
\hline Segurança Alimentar & 66 & 11,8 & $-0,65$ & 1,35 & \\
\hline IA leve & 133 & 23,9 & $-0,80$ & 1,10 & \\
\hline IA moderada & 226 & 40,5 & $-1,00$ & 1,07 & \\
\hline IA grave & 133 & 23,8 & $-1,11$ & 1,01 & 0,02 \\
\hline \multicolumn{6}{|l|}{ Área Geográfica } \\
\hline Zona Urbana & 302 & 54,1 & $-0,81$ & 1,13 & \\
\hline Zona Rural & 256 & 45,9 & $-1,06$ & 1,05 & 0,008 \\
\hline \multicolumn{6}{|l|}{ Renda "per capita" } \\
\hline$<0,25$ & 304 & 56,7 & $-1,06$ & 0,99 & \\
\hline $0,25-0,49$ & 159 & 29,7 & $-0,87$ & 1,16 & \\
\hline $0,50-0,74$ & 43 & 8,0 & $-1,04$ & 1,29 & \\
\hline$\geq 0,75$ & 30 & 5,6 & $-0,32$ & 0,97 & 0,002 \\
\hline \multicolumn{6}{|l|}{ Escolaridade materna } \\
\hline Nunca freqüentou escola & 21 & 3,9 & $-1,21$ & 0,85 & $<0,001$ \\
\hline $1-4$ anos & 285 & 53,4 & $-1,07$ & 1,04 & \\
\hline$>4$ anos & 228 & 42,7 & $-0,71$ & 1,19 & \\
\hline \multicolumn{6}{|l|}{ Sexo } \\
\hline Masculino & 311 & 55,7 & $-0,93$ & 1,09 & \\
\hline Feminino & 247 & 44,3 & $-0,92$ & 1,12 & 0,90 \\
\hline \multicolumn{6}{|l|}{ Idade } \\
\hline$<24$ meses & 216 & 38,7 & $-0,84$ & 1,25 & \\
\hline$\geq 24$ meses & 342 & 61,3 & $-0,98$ & 1,00 & 0,16 \\
\hline
\end{tabular}

materna continuou significante após ajuste para a variável renda familiar per capita, enquanto a localização geográfica deixou de ser significante. No modelo 3, o efeito da (in)segurança alimentar não apresentou significância estatística após ajuste para as variáveis dos blocos 1 e 2. Por fim, a entrada da idade, no modelo 4 , não se revelou significante com a resposta do estado nutricional, após ajuste para as demais variáveis.

\section{Discussão}

Preliminarmente, convém observar que os resultados apresentaram uma consistência interna elevada, visto que o Alpha de Cronbach foi de 0,91, demonstrando que as perguntas foram respondidas de acordo com a expectativa teórica própria da escala, em ordem crescente de gravidade da insegurança alimentar.

Os resultados deste trabalho revelam que a prevalência da condição de insegurança alimentar foi semelhante aos encontrados por Pereira et al $2006^{26}$, ao estudarem a situação alimentar em região de alta vulnerabilidade social da cidade de São Paulo $(88,0 \%)$. No entanto, nossos resultados foram bem superiores aos verificados na Região Nordeste, a partir da Pesquisa Nacional por Amostra de Domicílios - PNAD ${ }^{17}$ (2004) e da Pesquisa Nacional de Demografia e Saúde - PNDS $^{27}$ (2006), onde, respectivamente, $59,1 \%$ e $52,9 \%$ das famílias foram classificadas na categoria de insegurança alimentar. Ademais, também se mostram 
Tabela 2 - Regressão linear hierarquizada entre os determinantes do índice estatura-para-idade de crianças menores de 5 anos - São João do Tigre, 2005.

Table 2 - Hierarchical linear regression analysis of the determinants of the height-for-age index of children under five years of age. São João do Tigre, 2005.

\begin{tabular}{|c|c|c|c|c|c|}
\hline Variáveis & $\begin{array}{c}\text { Modelo } 1 \\
\beta\end{array}$ & $\begin{array}{c}\text { Modelo } 2 \\
\beta\end{array}$ & $\begin{array}{c}\text { Modelo } 3 \\
\beta\end{array}$ & $\begin{array}{c}\text { Modelo } 4 \\
\beta\end{array}$ & $\mathbf{R}^{2}(\%)$ \\
\hline \multicolumn{6}{|c|}{ Renda familiar per capita (SM) } \\
\hline$<0,25$ & $-0,23^{*}$ & $-0,12$ & $-0,07$ & $-0,07$ & $11,0(11,0)$ \\
\hline \multicolumn{6}{|c|}{ Escolaridade Materna' ${ }^{1}$} \\
\hline $0-4$ anos & & $-0,29 * *$ & $-0,23^{*}$ & $-0,22^{*}$ & \\
\hline \multicolumn{6}{|c|}{ Área geográfica ${ }^{1}$} \\
\hline Rural & & $-0,14$ & $-0,14$ & $-0,13$ & $21,0(32,0)$ \\
\hline \multicolumn{6}{|c|}{ (In)Segurança ${ }^{2}$ Alimentar } \\
\hline IA Leve & & & $-0,07$ & $-0,08$ & \\
\hline IA Moderada & & & $-0,17$ & $-0,18$ & \\
\hline IA Grave & & & $-0,23$ & $-0,24$ & $2,0(34,0)$ \\
\hline \multicolumn{6}{|l|}{ Idade $^{3}$} \\
\hline$\geq 24$ meses & & & & $-0,08$ & $1,0(35,0)$ \\
\hline
\end{tabular}

$\beta$ Coeficiente de regressão. $\beta$ Regression coefficient

$\mathrm{R}^{2}$ Coeficiente de determinação. $R^{2}$ Determination coefficient

SM: salário mínimo; IA: insegurança alimentar. SM: minimum wages; Al: food insecurity

${ }^{1}$ Ajustado pela variável renda familiar per capita. ${ }^{1}$ Adjusted for per capita family income

${ }^{2}$ Ajustado pela variável renda familiar per capita, escolaridade materna e área geográfica. ${ }^{2}$ Adjusted for per capita family income, maternal education and geographic area

${ }^{3}$ Ajustado pela variável renda familiar per capita, escolaridade materna, área geográfica e (In)segurança alimentar. ${ }^{3}$ Adjusted for per capita family income, maternal education and geographic area and food (in)security.

Níveis de significância: ${ }^{*} p \leq 0,05 ; * * 0,01$. Significance level: * $p \leq 0.05 ; * * \leq 0.01$

bem acima das prevalências descritas por Vianna \& Segall-Corrêa ${ }^{28}$ (2008), ao analisarem a insegurança alimentar das famílias residentes em 14 municípios da interior do estado da Paraíba, onde 52,5\% da população foi classificada em diferentes graus de insegurança alimentar. Comprova-se, portanto, a condição de precariedade das condições de vida de São João do Tigre.

Por outra parte, a prevalência de insegurança alimentar mais elevada no meio rural, onde as formas moderada e grave se situaram, cumulativamente, em torno de $70 \%$, demarcam diferenças espaciais que concordam com as tendências encontradas na PNAD-2004 $4^{17}$ para o conjunto do país. Apesar de consideravelmente elevados, atingindo patamares raramente encontrados em outros estudos no Brasil, os resultados de São João do Tigre expressam experiências negativas acumuladas em sucessivas gerações que sobrevivem no limiar da pobreza absoluta, submetendo-se ao caráter aleatório e sazonal do emprego e renda nas localidades mais pobres do semi-árido.

Para entender o elevado grau de insegurança alimentar ( $87 \%$ de toda a amostra) e a predominância de formas moderadas e graves, representando $64 \%$ das famílias analisadas, é pertinente lembrar a própria intencionalidade do estudo, ao se escolher uma localidade que se distinguisse no quadro regional do Nordeste pela marcante precariedade de suas condições de vida. $\mathrm{O}$ semi-árido é o espaço geográfico mais pobre do Nordeste. A microrregião dos Cariris Velhos, compondo a vertente ocidental do altiplano da Borborema, configurava, no advento dos anos 2000, o subconjunto espacial de mais baixa renda do semi-árido paraibano. Mais ainda, São João do Tigre, 15 anos antes da realização da pesquisa, era considerado o município mais pobre de todo o Nordeste e do Brasil. Por outra 
parte, como se observou com o levantamento de dados primários da pesquisa, em 2005, 56,7\% das famílias tinham uma renda familiar per capita abaixo de 1/4 do salário mínimo, caracterizando uma situação de pobreza absoluta, enquanto $57,3 \%$ das mães apresentavam uma história de escolaridade formal até 4 anos de estudo, o que revela outra característica do grave déficit social de sua população.

Ao lado destes aspectos quantitativos, convém ressaltar, como área de interesse peculiar adotado na escolha deste município, a grande interdependência econômica entre o meio urbano e a zona rural, de tal modo que as contingências favoráveis ou adversas da situação no campo pudessem se refletir, de forma marcante e imediata, no próprio status de vida do meio urbano. Em outras palavras, que as crises periódicas e previsíveis das secas, desorganizando a já escassa produção agropecuária do campo, pudessem impactar negativamente o processo de interações socioeconômicas dos dois espaços, o urbano e o rural, ampliando o alcance e os efeitos populacionais da crise climática.

Assim, a aleatoriedade das chuvas, a baixa fertilidade natural dos solos, a precariedade dos recursos tecnológicos utilizados na produção agropecuária, a escassa disponibilidade de terras, num espaço em que mais de $3 / 4$ das propriedades têm menos de 20 hectares, tornam a economia extremamente vulnerável, definindo um quadro estrutural de permanente insegurança econômica e social. Dentro deste cenário, a própria insegurança alimentar, como instrumento de avaliação essencialmente subjetivo, passa a retratar as expectativas negativas do processo econômico e social, profundamente marcado pela chamada "cultura da pobreza": instabilidade de emprego e renda, desinformação, moradias precárias, escasso conhecimento das oportunidades e mecanismos do mercado, apelo às crenças religiosas de um determinismo sobrenatural dos problemas e respectivas soluções, apoio no paternalismo político e social de lideranças que exercem o poder político e econômico das comunidades mais pobres ${ }^{29}$. Dentro desse contexto, não surpreende a massificação da insegurança alimentar, que incorpora quase todo o universo populacional do município.

O que de fato parece surpreender nos resultados é a elevada prevalência de normalidade antropométrica das crianças, segundo os índices peso/idade e peso/altura (IMC), alcançando valores que seriam aceitáveis como normais de acordo com o próprio padrão internacional de referência ${ }^{30}$, o que seria, em princípio, um contra-senso epidemiológico: a coexistência de um risco praticamente generalizado de insegurança alimentar com um quadro excepcionalmente favorável de normalidade antropométrica nas crianças. Aliás, este aparente paradoxo já foi assinalado a partir de resultados recentes, caracterizando um momento peculiar do rápido processo de transição epidemiológica que ocorre no Brasil ${ }^{31}$. A mesma situação não se repete quando se considera o índice estatura/idade: em São João do Tigre, o déficit estatural das crianças seria quase duas vezes (população urbana) ou três vezes (população rural) maior que as prevalências mais recentes detectadas no Brasil, e bem acima dos resultados do Nordeste $^{27}$ e da própria zona semi-árida ${ }^{32}$.

O segundo aspecto, também um tanto surpreendente em termos conceituais, consiste no grande desencontro entre a prevalência de insegurança alimentar como regra e a baixa ocorrência de desnutrição que, comparativamente, aparece como uma exceção (caso dos índices P/I e P/E), até mesmo da relação estatura/idade. $\mathrm{Na}$ realidade, em relação ao retardo do crescimento estatural, único indicador que daria "visibilidade epidemiológica" ao problema residual da desnutrição, é interessante observar que, mesmo no grupo de segurança alimentar, a média negativa $(-0,65$ escoresZ) na relação estatura/idade expressa um comprometimento importante do atraso do crescimento, enquanto na condição mais grave de insegurança alimentar este valor se situava em -1,11 escores-Z. Isto indicaria que, apesar da diferença estatisticamente 
significativa, a variação de estado nutricional não apresenta a correlação que, em termos conceituais, seria de se esperar entre os resultados da avaliação da (in)segurança alimentar com a classificação do estado de nutrição.

$\mathrm{Na}$ realidade, tanto a questão da segurança alimentar como a avaliação do estado nutricional por um indicador "histórico" (no sentido em que representa mais o passado do crescimento linear, acumulando déficits e avanços) devem ser contextualizadas no processo complexo do desenvolvimento dos indivíduos e da comunidade, e não apenas no retrato instantâneo dos estudos de prevalência. No semi-árido do Nordeste, como em todas as regiões de graves e repetidas crises alimentares no mundo, a experiência dramática da fome deixa memórias traumáticas e duradouras, que se colocam como ameaças permanentes para as pessoas atingidas. Estas impressões negativas se manifestam claramente nos inquéritos de caráter subjetivo, como o questionário de Cornell ${ }^{13,14}$ ou a Escala Brasileira de Insegurança Alimentar, independente da condição nutricional em si e, portanto, mais dependente da vulnerabilidade econômica e social das famílias. Esta perspectiva, que compete mais à antropologia e à psicologia social, explicaria porque, na análise de regressão linear, apenas a renda familiar, no modelo 1, e a baixa escolaridade, em toda a sequência de modelos, se mantiveram significativos na explicação do índice estatura/ idade. Convém ressaltar o estudo de Hoff$\operatorname{man}^{33}$ (2008), ao analisar os determinantes da insegurança alimentar no Brasil a partir dos dados da PNAD de 2004, verificando que o fator mais importante da insegurança alimentar foi, sem dúvida, a baixa renda familiar per capita, que já se torna, por si, uma das condições básicas da insegurança. Isto ajudaria a entender porque, apesar de sua frequência e de sua gravidade, a insegurança alimentar não se manteve no modelo explicativo.

Finalmente, prevalece a conclusão de que, mesmo em população de elevado grau de pobreza, baixo Índice de Desenvolvimento Humano (IDH de São João do Tigre: $0,527)^{34}$ e prevalência quase generalizada de insegurança alimentar, os indicadores antropométricos do estado nutricional podem revelar uma situação bem mais favorável, até mesmo imprevista, constituindo um aparente paradoxo ${ }^{31}$. Portanto, não pode ser interpretada apenas no campo da validade interna dos resultados em si, mas como parte de um processo de rápidas mudanças que caracteriza a transição nutricional em curso, atingindo, inclusive, as populações de condições de vida excepcionalmente desfavoráveis.

\section{Referências}

1. Valente FLS. Fome, desnutrição e cidadania: inclusão social e direitos humanos. Saúde Sociedade, 2003; 12: 5160 .

2. Maluf RSJ. Segurança alimentar e nutricional. Petrópolis: Vozes; 2007.

3. Salles CR; Moura JKB; Medeiros PRM; Escoda MSQ. Risco e Segurança Alimentar, 2001. Disponível em http://www.ufrnet.br/ scorpius/391-Risco\%20e\%20 Planej\%20Alim.htm\# PLANEJAMENTO\%20ALIMENTAR [Acessado em 28 de novembro de 2006].

4. Freitas MCS, Pena PGL. Segurança alimentar e nutricional: a produção do conhecimento com ênfase nos aspectod da cultura. Rev Nutr 2007; 20(1): 69-81.
5. Panigassi G. Inquérito populacional sobre a percepção da segurança alimentar intrafamiliar no município de Campinas, SP [tese de doutorado]. Campinas: Universidade Estadual de Campinas; 2005. Disponível em http://libdigi.unicamp.br/ document/?code=vtls000389627 [Acessado em 1 de novembro de 2007].

6. FAO (Food and Agriculture Organization) (Organización de las Naciones Unidas para la Agricultura y la Alimentación), 1974. Conferencia Mundial de los Alimentos. Roma: FAO.

7. OMS (Organización Mundial de la Salud), 1978. Atención Primaria de Salud. Genebra: OMS. (Serie Salud para Todos, 1). 
8. Segall-Corrêa AM. Insegurança alimentar medida a partir da percepção das pessoas. Estud av 2007; 21(60): 143-54.

9. Sícoli JL. Pactuando conceitos fundamentais para a construção de um sistema de monitoramento da SAN. Disponível em http://www.polis.org.br/download/65. pdf [Acessado em 21 de outubro de 2007].

10. Ruel MT. Is dietary diversity an indicator of food security or dietary quality? A review of measurement issues and research needs. Food Nutr Bull 2003; 242: 231-32.

11. Valente FLS. Do combate à fome à segurança alimentar e nutricional: o direito à alimentação adequada. $R$ Nutr Puccamp 1997; 10(1): 20-36.

12. Salles-Costa R. Avaliação alimentar em Duque de Caxias (RJ). Estud av 2006; 21(60): 135-42.

13. Radimer KL; Olson CM; Greene JC; Campbell CC; Habicht JP. Understanding hunger and developing indicators to assess it in women and children. J Nutr 1992; 24(1): 36-45.

14. Wehler CA, Scott RI, Anderson JJ. The Community Childhood identification Project: a model of domestic hunger-Demonstration project in Seattle, Washington. $J$ Nutr Educ 1992; 24: 29-35.

15. Bickel G, Nord M, Price C, Hamilton W, Cook J. Guide to measuring household food security: revised 2000. United States Department of Agriculture: Food and Nutrition Service; março de 2000.

16. Segall-Corrêa AM, Pérez-Escamilla R, Maranha LK, Sampaio MFA, Yuyama L, Alencar F et al. Projeto: Acompanhamento e avaliação da segurança alimentar de famílias brasileiras: validação de metodologia e de instrumento de coleta de informação. Campinas: Departamento de Medicina Preventiva e Social, Universidade Estadual de Campinas/Organização Pan-Americana da Saúde/ Ministério de Saúde; 2004 (Relatório Técnico).

17. Instituto Brasileiro de Geografia e Estatística (IBGE). Pesquisa Nacional por Amostra de Domicílios - PNAD. Segurança Alimentar: 2004. Rio de Janeiro; 2006.

18. Batista Filho M, Rissin A. A transição nutricional no Brasil: tendências regionais e temporais. Cad Saúde Pública 2003; 19(1): 181-91.

19. Messer E, Ross EM. Talking to patients about food insecurity. Nutr Clin Care 2002; 5: 168-81.

20. Rose D. Economic determinants and dietary consequences of food insecurity in the United States. $J$ Nutr 1999; 129: 517-20.

21. World Health Organization (WHO). Physical Status: The use and interpretation of anthropometry. Geneva; 1995. (WHO - Technical Report Series, 854).
22. World Health Organization (WHO). Anthro for personal computers, version 2, 2007: Software for assessing growth and development of the world's children. Geneva:WHO; 2007. Disponível em http://www.who. int/childgrowth/software/en/ [Acessado em $23 \mathrm{de}$ janeiro de 2007].

23. World Health Organization (WHO). Multicentre Growth Reference Study Group. WHO child growth standards based on length/height, weight and age. Acta Paediatr 2006; (S450): 76-85.

24. Ministério da Saúde. Coordenação Geral da Política de Alimentação e Nutrição. Vigilância Alimentar e Nutricional: SISVAN. Orientações para a coleta e análise de dados antropométricos em serviços de saúde. Norma Técnica - SISVAN. Material Preliminar. Brasília; 2008.

25. Monteiro CA, Conde WL. Tendência secular da desnutrição e da obesidade na infância na cidade de São Paulo (1974-1996). Rev Saúde Pública 2000; 34 (S6): 5261.

26. Pereira DA, Vieira VL, Fiore EG, Cervato-Mancuso AM. Insegurança Alimentar em Região de Alta Vulnerabilidade Social da cidade de São Paulo. Segurança Alimentar e Nutricional 2006; 13(2): 34-42.

27. Ministério da Saúde. Pesquisa Nacional de Demografia e Saúde da Criança e da Mulher - PNDS 2006. Relatório. Brasília; 2008.

28. Vianna RPT, Segall-Corrêa. Insegurança alimentar das famílias residentes em municípios do interior do estado da Paraíba, Brasil. Rev Nutr 2006; S21: 111-22.

29. Andrade MC. Nordeste Semi-Árido. Limitações e Potencialidades. In: Batista Filho M. (Org) Viabilização do Semi-Árido Nordestino. Série de Publicações Científicas do Instituto Materno Infantil de Pernambuco 2001; 6: 12-8.

30. Batista Filho M, Miglioli TC, Santos MC. Anthropometric normality in adults: the geographical and socioeconomic paradox of the nutritional transition in Brazil. Rev Bras Saúde Matern. Infant 2007; 7(4): 487-93.

31. Batista Filho M. Introdução. Cad Estud Desenv Soc Debate 2006; 4: 9-16.

32. Monteiro CA, Conde WL, Konno SC. Análise do Inquérito Chamada Nutricional 2005. Cad Estud Desenv Soc Debate 2006; 4: 29-36.

33. Hoffmann R. Determinantes da insegurança alimentar no Brasil. Análise dos dados da PNAD de 2004. Rev Segur Alim Nutr 2008; 15(1): 49-61.

34. Programa das Nações Unidas para o Desenvolvimento - PNUD. Atlas do Desenvolvimento Humano no Brasil. Disponível em http://www.pnud.org.br/rdh [Acessado em 20 de março de 2007].

Recebido em: 02/02/09

Versão final reapresentada em: 03/06/09

Aprovado em: 23/06/09 